\title{
7 Lokale Aushandlungen und transregionale Gegenbewegungen
}

An der Neu-Verräumlichung von Land sind nicht nur Akteur:innen aus Staat, Wirtschaft und Finanzindustrie beteiligt. Auch lokale Bevölkerungsgruppen nehmen entscheidenden Einfluss darauf, wie (und ob) Investitionsprojekte vor Ort umgesetzt werden und mit welchen sozio-ökonomischen und ökologischen Konsequenzen dies erfolgt. Gegen die oftmals existenzbedrohenden Auswirkungen des globalen Ansturms auf Land für Kleinbäuer:innen, Viehhirt:innen, Fischer:innen und indigene Bevölkerungsgruppen, vor allem im Globalen Süden, formierte sich in vielen betroffenen Regionen Widerstand. Das wohl berühmteste Beispiel sind die Massenproteste gegen den 2008 geschlossenen Pachtvertrag des südkoreanischen Konzerns Daewoo über 1,3 Millionen Hektar Ackerland in Madagaskar (etwa die Hälfte der landwirtschaftlichen Nutzfläche der Insel). Die Proteste führten nicht nur zur Annullierung des Vertrags, sondern gar zum Sturz des madagassischen Präsidenten. ${ }^{1}$ Mit Hilfe von Landbesetzungskampagnen protestierten auch ägyptische Bauernvereinigungen im Zuge des arabischen Frühlings gegen jahrzehntelange Prozesse der Landenteignung und Verdrängung. ${ }^{2}$ Und in Laos haben sich lokale Gemeinschaften der Landnahme durch chinesische Plantageninvestoren widersetzt, indem sie verwandtschaftliche, ethnische oder historische Bindungen zu verschiedenen Akteur:innen innerhalb des laotischen Staats mobilisierten. ${ }^{3}$ Dies sind nur einige Beispiele für die vielfältigen Taktiken des teils subtilen, teils offenen Widerstands, mit denen lokale Bevölkerungen gegen die aktuellen Neu-Verräumlichungen von Land mobilisieren - und diesen alternative Imaginationen und Verräumlichungsprojekte entgegensetzen. Diese beziehen sich auch auf breitere Themen wie ländliche Armut, indigene und kleinbäuerliche Rechte sowie Umweltverträglichkeit und Nachhaltigkeit. Lokale Aushandlungen sind jedoch keineswegs allein durch Widerstand und Protest charakterisiert. Auch hier muss räumlich und akteursspezifisch differenziert werden. Die oben beschriebenen Projekte der Neu-Verräumlichung von Land treten keineswegs immer als „externe“ Gewalt auf, die über das „Lokale“

1 D. Kress, Investitionen in den Hunger? Land Grabbing und Ernährungssicherheit in SubsaharaAfrika, Wiesbaden: Springer, 2012.

2 F. d. Lellis, „Peasants, Dispossession and Resistance in Egypt. An Analysis of Protest Movements and Organisations before and after the 2011 Uprising“, Review of African Political Economy 46 (2019) 162, S. 582-598.

3 M. Kenney-Lazar, „Governing Dispossession. Relational Land Grabbing in Laos“, Annals of the American Association of Geographers 108 (2018) 3, S. 679-694. 
hineinbricht. Vielmehr existieren vielschichtige Prozesse, die von der Hoffnung auf Teilhabe bis hin zu aktiver Partizipation lokaler Bevölkerungsgruppen reichen.

Die komplexen lokalen Aushandlungen der Neu-Verräumlichungen von Land stehen im Fokus dieses Abschnitts. Damit verbunden diskutieren wir die neue transregionale Dimension, die die aktuellen Widerstandsbewegungen auszeichnet. Widerstand gegen die Einhegung und Kommodifizierung von Land ist, historisch gesehen, nicht neu. Der Land Rush, so hebt Ouma hervor, weist nicht allein Parallelen zu den Einhegungen von Land während des europäischen Kolonialismus auf. Auch das Aufbegehren gegen diese ist ein regelmäßiger Bestandteil in der Geschichte des Kapitalismus. ${ }^{4}$ Wenn auch kein grundsätzlich neues Phänomen, so ist der Widerstand gegen großflächige Landkäufe doch neu ins Bewusstsein einer breiten und zunehmend globalen Öffentlichkeit gerückt. Dazu trägt auch bei, dass viele Gegenbewegungen heute nicht mehr ausschließlich lokal, sondern weltweit vernetzt agieren und ihren Protest in weithin sichtbaren, internationalen Foren wie den Vereinten Nationen artikulieren.

\subsection{Zwischen Widerstand und Einbindung}

Die griffige Formel von „Landnahme + Exklusion = Widerstand“, die zahlreiche Autor:innen im Zuge des Land Rush bemüht haben, greift zu kurz, um die komplexen Verräumlichungsprozesse $\mathrm{zu}$ beschreiben, die mit großflächigen Agrarinvestitionen einhergehen. Die lokale Neuaushandlung der Landfrage verlangt vielmehr nach einer differenzierten empirischen Beschreibung und theoretischen Einordnung der vielschichtigen lokalen Einbettungen großflächiger Agrarinvestitionen. Diese bewegen sich im Spannungsfeld zwischen der Mobilisierung von Widerstand gegen Verdrängung und Exklusion, der Forderung nach Teilhabe und Integration bis hin zur aktiven Partizipation und Unterstützung. Lokale Bevölkerungsgruppen sind weder passive Opfer noch kollektiv im Widerstand vereint, sondern durch heterogene Interessenlagen gekennzeichnet. ${ }^{5}$ Um die vielfältigen Neuaushandlungen der Landfrage theoretisch zu fassen, so argumentieren Borras und Franco, müssen die sich nach Klasse, Geschlecht, Generation, Ethnizität und

\footnotetext{
4 S. Ouma, „Land Grabbing“, in: N. Marquardt und V. Schreiber (Hrsg.), Ortsregister: Ein Glossar zu Räumen der Gegenwart, Bielefeld: Transcript, 2012, S. 171-177.

5 S. M. Borras und J. C. Franco, „Global Land Grabbing and Political Reactions ,From Below““, Third World Quarterly 34 (2013) 9, S. 1723-1747; R. Hall u. a., „Resistance, Acquiescence or Incorporation? An Introduction to ,Land Grabbing and Political Reactions from Below““, The Journal of Peasant Studies 42 (2015) 3-4, S. 467-488.
} 
Nationalität ausdifferenzierenden Interessenkonfigurationen in ihrer jeweiligen historisch-geografischen Spezifizität in den Blick genommen werden. ${ }^{6}$

Ein solchermaßen differenzierter Blick zeigt erstens, dass Konfliktlinien nicht nur zwischen lokalen (klein-)bäuerlichen Gemeinschaften und wirtschaftlichen oder staatlichen Eliten verlaufen, sondern auch innerhalb lokaler Bevölkerungsgruppen selbst. So zeigen zum Beispiel Fallstudien zu großflächigen Landkäufen auf dem Territorium der Gumuz in Äthiopien, wie lokale Chiefs in Absprache mit Regierungsvertreter:innen den Ausverkauf gemeinschaftlicher Agrarflächen zu ihrem eigenen Vorteil und gegen die Interessen ihrer eigenen Dorfgemeinschaften befördert haben. ${ }^{7}$ Gleichzeitig kann es lokalen Gemeinschaften auch gelingen, mächtige Interessengruppen in Wirtschaft und Regierung gegeneinander auszuspielen und somit ihren eigenen Forderungen Nachdruck zu verleihen. Ein prominentes Beispiel hierfür sind die zahlreichen Konflikte um Landzugang und -nutzung im ländlichen China. Um sich der vielfach illegalen Enteignung und Vertreibung durch lokale Behörden zu erwehren, appellierten Bäuer:innen in offenen und oftmals lautstarken Protesten an die Zentralregierung. Sie legitimierten ihr Anliegen, indem sie aufzeigten, wie Gesetze, Richtlinien und Werte des Staates von lokalen Behörden missachtet werden. ${ }^{8}$ Institutionalisierter Widerstand in dieser Form zieht seine Wirkungsmacht somit vor allem daraus, dass er konkurrierende Macht- und Interessenzentren innerhalb des Staates identifiziert und für die Durchsetzung eigener Ziele mobilisiert.

Lokale Reaktionen auf die zunehmende Konzentration von Agrarland in der Hand industrieller Agrarunternehmen und Finanzinvestoren sind zweitens nicht zwangsläufig von Widerstand und Aufbegehren geprägt. Im Gegenteil können auch Forderungen nach Inklusion und Teilhabe an den agro-industriellen Großprojekten überwiegen. Vor dem Hintergrund der globalen Krise der kleinbäuerlichen Landwirtschaft - geprägt von schrumpfenden Familienbetrieben, Perspektivlosigkeit und Mangel an Arbeitsplätzen - scheint für manche:n die Einbindung in agro-industrielle Großunternehmen und deren globale Warenketten - sei es als Vertragslandwirt oder als Landarbeiter - die bessere Alternative. So wurde für viele ländliche Räume der früheren Sowjetunion (wie der Ukraine oder der Schwarzerde-Region in Zentralrussland) das Fehlen lokaler Proteste gegen Prozesse der Landkonzentration und -kommodifizierung konstatiert. Mehr noch,

\footnotetext{
6 Ebd.

7 S. Moreda, „Listening to their Silence? The Political Reaction of Affected Communities to LargeScale Land Acquisitions. Insights from Ethiopia“, The Journal of Peasant Studies 42 (2015) 3-4, S. 517-539.

8 K. J. O’Brien und L. Li, Rightful Resistance in Rural China, Cambridge: Cambridge University Press, 2006.
} 
lokale Bevölkerungen artikulieren hier den expliziten Wunsch nach einer stärkeren Präsenz privatwirtschaftlicher und staatlicher Investoren in Land und Landwirtschaft, wie Vorbrugg zeigt. ${ }^{9}$ Er erklärt diesen Befund durch den Zerfall und die Abwertung der regionalen Landwirtschaft während des Niedergangs der Sowjetunion, welche die Handlungsoptionen ländlicher Bevölkerungen drastisch eingeschränkt hätten. ${ }^{10}$ Die im Zuge des Land Rush entstandenen Agrarunternehmen würden von der ländlichen Bevölkerung als Fortschreibung sowjetischer Kollektiv- und Staatsbetriebe wahrgenommen. Viele lokale Bewohner:innen zögen ein gesichertes Lohn- und Beschäftigungsverhältnis in einem solchen Großbetrieb den prekären wirtschaftlichen Bedingungen der Familienlandwirtschaft vor, so Vorbruggs Diagnose.

Beispiele eines Reisanbau- und Bewässerungsprojekts in Mali ${ }^{11}$ oder der Fall einer 11.000 Hektar umfassenden Zuckerrohrplantage in den nördlichen Philippinen $^{12}$ zeigen, dass lokale Bevölkerungen auch in anderen Regionen mit der Forderung nach Inklusion auf großflächige Landinvestitionen reagiert haben. Sie versuchten ihre Teilhabe an Investitionsprojekten zu sichern, indem sie Allianzen mit Regierungsbehörden und Vertreter:innen von Agrarunternehmen und Investoren schlossen, Land an Agrarunternehmen verpachteten, für diese Vertragslandwirtschaft betrieben oder als Plantagenarbeiter arbeiteten. Forschungen in Regionen mit einer hochindustrialisierten Landwirtschaft wie Australien haben darüber hinaus gezeigt, dass landwirtschaftliche Investitionen auch begrüßt werden können. Bedenken manifestierten sich dort vielmehr gegenüber spezifischen Akteuren und deren Investmentpraktiken oder -motiven. ${ }^{13}$ Auch positionieren sich seit einigen Jahren besonders unternehmerisch gesinnte Landwirt: innen als ,ideale Partner“ für transnational agierende Pensionsfonds und Private

\footnotetext{
9 A. Vorbrugg, „Not About Land, Not Quite a Grab. Dispersed Dispossession in Rural Russia“, Antipode 51 (2019) 3, S. 1011-1031; N. Mamonova, „Resistance or Adaptation? Ukrainian Peasants’ Responses to Large-Scale Land Acquisitions“, The Journal of Peasant Studies 42 (2015) 3-4, S. 607-634.

10 Vorbrugg, „Not About Land, Not Quite a Grab“.

11 N. Larder, „Space for Pluralism? Examining the Malibya Land Grab“, The Journal of Peasant Studies 42 (2015) 3-4, S. 839-858.

12 J. C. Franco, D. Carranza und J. Fernandez, New Biofuel Project in Isabela. Boon or Bane for Local People?, Amsterdam: Transnational Institute, 2011.

13 S. R. Sippel, N. Larder und G. Lawrence, „Grounding the Financialization of Farmland. Perspectives on Financial Actors as New Land Owners in Rural Australia“, Agriculture and Human Values 34 (2017) 2, S. 251-265.
} 
Equity Unternehmen, die auf der Suche nach Vermögensanlagen in Land und Landwirtschaft sind. ${ }^{14}$

Ob und unter welchen Bedingungen lokale Bevölkerungsgruppen tatsächlich von der Inklusion in Landinvestitionsprojekte profitieren können, ist dabei fraglich. Agroindustrielle Großplantagen bieten häufig nur ungenügende Arbeitsplatz- und Einkommensmöglichkeiten, während für die in Form von Vertragslandwirtschaft eingebundenen Kleinbäuer:innen neue Abhängigkeiten und Risiken entstehen. ${ }^{15}$ Und auch in Kontexten des Globalen Nordens sollte die sich in der Figur des farmer-investors manifestierende neue Symbiose von Finanzkapital und Landwirtschaft nicht darüber hinwegtäuschen, dass viele landwirtschaftliche Familienbetriebe auch hier durch die fortschreitende Finanzialisierung der Landwirtschaft in ihrer Existenz bedroht sind. Dennoch zeigen die vielfältigen politischen Reaktionen auf den globalen Land Rush, dass die Annahme, lokale Bevölkerungsgruppen lehnten sich grundsätzlich gegen die zunehmende Kommodifizierung und Finanzialisierung von Agrarland auf, zu kurz greift.

\subsection{Transnationaler Aktivismus und Land Grabbing als politischer Streitbegriff}

Die bisher beschriebenen Beispiele verweisen auf eine weitere sozial-räumliche Besonderheit aktueller Landtransformationen: Aushandlungsprozesse und politische Auseinandersetzungen um landbezogene Zugangs-, Nutzungs- und Eigentumsrechte artikulieren sich auf verschiedenen räumlichen Maßstabsebenen zugleich - von der Ebene der Dorfgemeinschaft über die Interaktion mit nationalen Regierungsvertreter:innen bis hin zu internationalen Foren wie der Nahrungsmittelorganisation der Vereinten Nationen (Food and Agriculture Organization, FAO). Hierbei kommt der Formierung neuer transnationaler Agrarbewegungen, die auf internationaler Ebene für Land- und Ernährungsrechte von Kleinbäuer:innen und indigenen Gruppen streiten, eine besondere Bedeutung zu. Prominenteste Vertreterin der sich um Landfragen formierenden transnationalen Agrarbewegungen ist La Via Campesina. Die seit 1993 aktive transnationale Bauernbewegung ist vor allem in Lateinamerika, Süd- und Südostasien, Westeuropa sowie in einigen Teilen Afrikas

14 Ebd.; A. Langford, „Capitalising the Farm Family Entrepreneur. Negotiating Private Equity Partnerships in Australia“, Australian Geographer 50 (2019) 4, S. $473-491$.

15 Siehe u. a. S. R. Sippel, Export(t)räume. Bruchzonen marokkanischer Landwirtschaft, Bielefeld: Transkript, 2014. 
vertreten und repräsentiert Millionen Kleinbäuer:innen, Landarbeiter:innen, Landlose und indigene Bevölkerungsgruppen in über 80 Ländern. ${ }^{16}$ La Via Campesina war nicht nur maßgelblich an der Erarbeitung einer 2018 verabschiedeten UN-Erklärung für die Rechte von Kleinbäuer:innen beteiligt, sondern streitet auch im Rahmen einer globalen „Kampagne für Agrarreform“ für eine gerechte Verteilung von Land und die Stärkung der bäuerlichen Landwirtschaft weltweit.

In Zusammenarbeit mit NROs wie GRAIN, dem Food First International Action Network (FIAN) sowie aktivistischen Wissenschaftler:innen haben transnationale Agrarbewegungen wie La Via Campesina entscheidend dazu beigetragen, die Landfrage unter dem politischen Kampfbegriff des Land Grabbing erneut in den internationalen Fokus zu rücken und den Druck der Zivilgesellschaft auf Wirtschaft und Staat zu erhöhen. Entgegen den marktaffinen Reformbestrebungen von Entwicklungsorganisationen wie der Weltbank, die individualisierte Eigentumsrechte (statt informellem Gemeinschaftsrecht) befürworten, geht es transnationalen Agrarbewegungen um eine Demokratisierung des Zugangs zu Land. Der Vision der Land- und Ernährungssouveränität entsprechend sollen Bäuer: innen direkt in Entscheidungsprozesse über die Nutzung und den Zugang zu Land mit einbezogen werden, wobei Fragen nach sozialer Gerechtigkeit und ökologischer Nachhaltigkeit im Vordergrund stehen. ${ }^{17}$

Die prominente Rolle, die transnationalem Aktivismus bei dieser Neuaushandlung von Land zukommt, ist nicht zuletzt der zunehmenden Auslagerung politischer Entscheidungsprozesse von der nationalen auf die internationale Ebene zuzuschreiben. Dieser Rescaling-Prozess wurde in den letzten Jahrzehnten von internationalen Institutionen angesichts einer beständig fortschreitenden globalen Verflechtung von Wirtschafts- und Finanzinteressen aktiv vorangetrieben. ${ }^{18}$ Verschiedene Organisationen wie die Weltbank, die Vereinten Nationen und die FAO nehmen heute aktiv Einfluss auf die Gestaltung von Landpolitik innerhalb spezifischer nationaler Kontexte. Dies verlangt auch von zivilgesellschaftlichen Akteuren wie NROs, Aktivist:innen und Agrarbewegungen, sich bei der Neuaushandlung der Landfrage nationen- und ebenenübergreifend zu organisieren und die Belange lokaler, von Landnutzungswandel betroffener Bevölkerungsgruppen auf der internationalen politischen Agenda zu positionieren.

In diesen Zusammenhang ist nicht zuletzt die enge Verflechtung zwischen transnationalem Aktivismus und akademischer Debatte einzuordnen. So wurden

16 La Via Campesina, International Peasants’ Movement, https://viacampesina.org/en/ (Zugriff 8. November 2020).

17 S. M. Borras, J. C. Franco und S. M. Suárez, „Land and Food Sovereignty“, Third World Quarterly 36 (2015) 3, S. $600-617$.

18 D. Hall, Land, Cambridge: Polity, 2013. 
politische Streitbegriffe wie Land Grabbing von der akademischen Forschung zur Transformation von Landnutzungs- und Besitzrechten aufgegriffen und für politische Zwecke mobilisiert. Zahlreiche Konferenzen an der Schnittstelle zwischen Aktivismus und Wissenschaft, die sich mit Konflikten um großflächigen Landnutzungswandel beschäftigt haben, zeigen, wie sich Aktivismus und Wissenschaft zu neuen Formen aktivistischer Wissenschaft oder wissenschaftlichem Aktivismus zusammenschließen. Auch wenn Forscher:innen dieser Verquickung nicht unkritisch gegenüberstehen bzw. diese kontinuierlich reflexiv begleiten, ${ }^{19}$ wurde mit dem aktivistischen Begriff des Land Grabbing ein wirkmächtiger Interpretationsrahmen in der kritischen Agrarforschung geschaffen, dessen oftmals unausgesprochene Annahmen die wissenschaftliche Debatte stark geprägt haben. Dies zeigt sich am starken Fokus der Debatte auf den Globalen Süden und der Betonung von (gewaltvollem) Konflikt und Widerstand. „Alltägliche“ und weniger spektakuläre Formen der Verdrängung ${ }^{20}$ wurden dadurch aber auch unsichtbar gemacht und Erfahrungs- und Deutungsrahmen lokaler Bevölkerungsgruppen oftmals überformt. Weder ist Land Grabbing für alle Situationen und Kontexte zwangsläufig die richtige Diagnose, noch ist die Verteidigung der „bäuerlichen Lebensweise“ ein von allen Akteur:innen notwendig angestrebtes Ziel. $^{21}$

19 Eine kritische Auseinandersetzung findet sich früh bei M. Edelman, C. Oya und S. M. Borras, „Global Land Grabs. Historical Processes, Theoretical and Methodological Implications, and Current Trajectories“, Third World Quarterly 34 (2013) 9, S. 1517-1531; C. Oya, „Methodological Reflections on ,Land Grab' Databases and the ,Land Grab' Literature ,Rush'“, The Journal of Peasant Studies 40 (2013) 3, S. 503-520; sowie I. Scoones u. a., „The Politics of Evidence“. Siehe auch Schoenberger, Hall und Vandergeest, „What happened when the Land Grab came to Southeast Asia?“.

20 Vorbrugg, „Not About Land, Not Quite a Grab“.

21 Larder, „Space for Pluralism?“; Mamonova, „Resistance or Adaptation?“. 